\title{
Adherence to guidelines: primary prevention with aspirin in 1125 medical check-up participants
}

\author{
Jin Hee Im¹, Sang Won Han', Seon Yeong Lee², Jong Sam Baik \\ 'Department of Neurology, Sanggye Paik Hospital, Inje University College of Medicine, Seoul 01757, South Korea. \\ ${ }^{2}$ Department of Family Medicine, Sanggye Paik Hospital, Inje University College of Medicine, Seoul 01757, South Korea.
}

Correspondence to: Dr. Jong Sam Baik, Department of Neurology, Sanggye Paik Hospital, Inje University College of Medicine, 761-1 Sanggye 7-dong, Nowon-gu, Seoul 01757, South Korea. E-mail: jsbaik@paik.ac.kr

How to cite this article: Im JH, Han SW, Lee SY, Baik JS. Adherence to guidelines: primary prevention with aspirin in 1125 medical check-up participants. Vesse/ Plus 2018;2:5. http://dx.doi.org/10.20517/2574-1209.2018.07

Received: 5 Mar 2018 First Decision: 22 Mar 2018 Revised: 4 Apr 2018 Accepted: 11 Apr 2018 Published: 18 Apr 2018

Science Editor: Aaron S. Dumont Copy Editor: Jun-Yao Li Production Editor: Cai-Hong Wang

\begin{abstract}
Aim: The aim of the present study was to assess the 10-year cardiovascular disease (CVD) risk and to apply the current recommendations on aspirin use for primary prevention in Korean participants undergoing a medical check-up.
\end{abstract}

Methods: Adults aged 50 to 69 years were eligible for the study if they did not have a history of atherosclerotic CVD (ASCVD) or stroke. The 10-year CVD risk was calculated using the ASCVD risk estimator (http://tools.acc.org/ ASCVD-Risk-Estimator).

Results: A total of 1125 participants were enrolled in this study. The mean age was 57 years, and 32\% of the participants were women. Based on the 2016 US Preventive Services Task Force recommendations, aspirin was indicated in 266 (23.6\%) participants but only 44 (3.9\%) participants were taking aspirin regularly. Among these participants, aspirin was prescribed appropriately in $36 \%$ of the participants, suggesting that only $6 \%$ of the participants were taking aspirin appropriately and 3.3\% of the participants were taking aspirin inappropriately. Logistic regression analysis showed that treatment for hypertension was significantly associated with taking aspirin (odds ratio 7.49; 95\% confidence interval 3.62-15.49).

Conclusion: Our study suggested that there may be an opportunity for decreasing the rate of CVD as well as the risk for major bleeds through tailored education on aspirin use.

Keywords: Aspirin, cardiovascular disease, guideline, primary prevention, stroke 


\section{INTRODUCTION}

Aspirin provides the benefit of primary prevention of vascular events in men or women whose risk for myocardial infarction (MI) or ischemic stroke, respectively, is high enough to outweigh the risk for bleeding ${ }^{[1]}$. In patients with atherosclerotic vascular disease, long-term antiplatelet therapy reduces the risk of vascular events. The benefits of long-term antiplatelet therapy substantially exceed the bleeding risk. For primary prevention of vascular events, aspirin is frequently taken regularly with or without a doctor's prescription. In such cases, the balance is less clear because the risks without aspirin and the benefits of aspirin are generally an order of magnitude lower than in secondary prevention ${ }^{[2-4]}$. In a collaborative meta-analysis, the use of aspirin provided a $12 \%$ proportional reduction in serious vascular events, due mainly to a reduction in nonfatal MI, and the net effect on stroke was not significant ${ }^{[5]}$. In 2016, the US Preventive Services Task Force (USPSTF) updated the recommendations on low-dose aspirin use for primary prevention of cardiovascular disease (CVD) and colorectal cancer (CRC), based on the American College of Cardiology/American Heart Association (ACC/AHA) risk calculator ${ }^{[4]}$. Prevention of CVD events is important, and understanding the physician's recommendations for aspirin use is essential for the management of quality of health care. The aim of the present study was to assess the 10-year CVD risk and to apply the current recommendations on aspirin use for primary prevention in Korean participants undergoing a medical check-up.

\section{METHODS}

\section{Participants}

Between January 2014 and December 2016, the participants who underwent a medical check-up at Sanggye Paik Hospital Health Promotion Center were enrolled into this study. Adults aged 50 to 69 years were eligible for the study if they did not have a history of atherosclerotic cardiovascular disease (ASCVD) or stroke with a low density lipoprotein (LDL)-cholesterol $<190 \mathrm{mg} / \mathrm{dL}$ and if they agreed to provide informed consent. They were classified as having an ASCVD history when they reported a history of MI, coronary bypass, or angioplasty. The participants' demographics, vascular risk factors, and medication history were collected at baseline. The medications taken regularly during the month preceding their visit were recorded. Physical examination, including blood pressure measurements, electrocardiogram, and blood testing were also performed.

The 2016 USPSTF recommendations on low-dose aspirin use for primary prevention of CVD and CRC are as follows: adults aged 50 to 69 years who have $\geq 10 \%$ 10-year CVD risk, are willing to take low-dose aspirin daily and have a life expectancy of at least 10 years, and are not at an increased risk for bleeding. With respect to adults aged 60 to 69 years, the decision to initiate aspirin use should be an individual one. The evidence of aspirin use in adults younger than 50 years and aged 70 years or older is insufficient ${ }^{[4]}$. The 10-year CVD risk was calculated using the ASCVD risk estimator (http://tools.acc.org/ASCVD-Risk-Estimator). The risk factors for the ACC/AHA ASCVD risk calculation were gender, age, race, total cholesterol, high density lipoprotein (HDL)-cholesterol, diabetes, treatment for hypertension, systolic blood pressure (SBP), and cigarette smoking. In this study, hypertension was defined as a SBP of at least $140 \mathrm{mmHg}$ or a diastolic blood pressure (DBP) of at least $90 \mathrm{mmHg}$. Diabetes mellitus was defined as present if the participant was receiving hypoglycemic agents or the fasting serum glucose level was $126 \mathrm{mg} / \mathrm{dL}$ or higher ${ }^{[6]}$. Participants who smoked regularly during the previous year were classified as current smokers.

High-resolution B-mode ultrasound measurements were performed in some participants according to the guidelines of the Mannheim intima-media thickness (IMT) Consensus ${ }^{[7]}$. The methods of IMT measurement have been published previously ${ }^{[s]}$. In brief, a single longitudinal lateral image of bilateral common carotid arteries (CCA) was obtained proximal to the carotid bulb, with the patient in the supine position. Automatic measurements of the CCA-IMT were performed approximately at $10 \mathrm{~mm}$ proximal to the carotid bulb. In this study, mean IMT of both carotid arteries was used for analysis. 
Table 1. Baseline demographic and clinical characteristics of participants

\begin{tabular}{lllll}
\hline Characteristics & Total $(\boldsymbol{n}=\mathbf{1 1 2 5})$ & Men $(\boldsymbol{n}=\mathbf{7 6 4})$ & Women $(\boldsymbol{n}=\mathbf{3 6 1})$ & $\boldsymbol{P}$ value \\
\hline Age (years) & $57.1(4.86)$ & $56.7(4.66)$ & $57.7(5.18)$ & 0.001 \\
SBP (mmHg) & $121.6(14.33)$ & $123.0(13.84)$ & $118.8(14.96)$ & $<0.0001$ \\
DBP (mmHg) & $77.7(10.78)$ & $79.3(10.53)$ & $74.3(10.50)$ & $<0.0001$ \\
Hypertension & $316(28.1)$ & $228(29.8)$ & $88(24.4)$ & 0.057 \\
Diabetes mellitus & $111(9.9)$ & $85(11.1)$ & $26(7.2)$ & 0.039 \\
Dyslipidemia & $256(22.8)$ & $169(22.1)$ & $87(24.1)$ & 0.460 \\
Current smoking & $230(20.4)$ & $222(29.1)$ & $8(2.2)$ & $<0.0001$ \\
Total cholesterol (mg/dL) & $192.8(33.21)$ & $189.8(32.93)$ & $199.1(32.97)$ & $<0.0001$ \\
LDL cholesterol (mg/dL) & $119.9(24.02)$ & $118.8(24.04)$ & $122.3(23.84)$ & 0.021 \\
HDL cholesterol (mg/dL) & $50.4(11.79)$ & $47.9(10.35)$ & $55.6(12.87)$ & $<0.0001$ \\
Regular alcohol drinking & $669(59.5)$ & $559(73.2)$ & $110(30.5)$ & $<0.0001$ \\
Regular physical activity & $642(57.1)$ & $454(59.4)$ & $188(52.1)$ & $<0.0001$ \\
Regular antihypertensive therapy & $282(25.1)$ & $202(26.4)$ & $80(22.2)$ & 0.122 \\
Current aspirin use & $44(3.9)$ & $34(4.5)$ & $10(2.8)$ & 0.175 \\
\hline
\end{tabular}

Data are means (SD) or numbers (\%). SBP: systolic blood pressure; DBP: diastolic blood pressure; LDL: low-density lipoprotein; HDL: high-density lipoprotein

All study participants provided informed consent, and the study design was approved by the appropriate ethics review board and conducted in accordance with the Good Clinical Practice guidelines and the Declaration of Helsinki.

\section{Statistical analysis}

Data are expressed as mean (standard deviation) or number (percent). The baseline characteristics of the two groups were compared by a Student $t$ test for continuous variables and by a $\chi^{2}$ test for categorical variables. Logistic regression models were used to analyze the relationship between ASCVD risk calculation factors and taking aspirin. Due to this trial enrolled single ethnicity, gender, age, total cholesterol, HDL-cholesterol, diabetes, treatment for hypertension, SBP, and cigarette smoking were used as variables for the study. Twosided null hypotheses of no difference were rejected at $P<0.05$. SAS version 4.2 software (SAS Institute Inc, Cary, NC, USA) was used for statistical analysis.

\section{RESULTS}

During the study period, a total of 1125 participants were enrolled in this study. Table 1 shows the baseline characteristics of the enrolled participants. The mean age was 57 years, and $32 \%$ of the participants were women. With respect to clinical history and vascular risk factors, $28 \%$ of the participants had a history of hypertension, $10 \%$ of the participants had diabetes, $23 \%$ of the participants had dyslipidemia, and $20 \%$ of the participants were current tobacco smokers. The SBP and DBP were significantly higher in men. The frequency of diabetes, smoking, and regular alcohol drinking/physical activity was also higher in men, whereas age, total, LDL and HDL-cholesterol levels were higher in women.

Based on the 2016 USPSTF recommendations, aspirin was indicated in 266 (23.6\%) participants but only 44 (3.9\%) participants were taking aspirin regularly [Table 2]. Among these participants, aspirin was prescribed appropriately in $36 \%$ of the participants, suggesting that only $6 \%$ of the participants were taking aspirin appropriately and $3.3 \%$ of the participants were taking aspirin inappropriately. Table 3 demonstrates the 10 -year CVD risk and the aspirin use according to gender and age. Among men, 253 (33.1\%) participants had $\geq 10 \%$ 10-year CVD risk [Figure 1]. Aspirin was recommended in $20.1 \%$ of the male participants aged 50-59 years and in $75.1 \%$ of the male participants aged 60-69 years. Among these male participants, however, only $15(5.9 \%)$ were taking aspirin appropriately and 3.7\% were taking aspirin inappropriately. Among women, 13 (3.6\%) participants had $\geq 10 \%$ 10-year CVD risk and none of the participants were taking aspirin appropriately. Aspirin was recommended only in 9.8\% of the female participants aged 60-69 years [Table 3]. 
Table 2. The 10-year CVD risk and aspirin use

\begin{tabular}{llll}
\hline & Total $(\boldsymbol{n}=\mathbf{1 1 2 5})$ & Men $(\boldsymbol{n}=\mathbf{7 6 4})$ & Women $(\boldsymbol{n}=\mathbf{3 6 1})$ \\
\hline $10-y e a r$ CVD risk (\%) & $0.5-46.2$ & $1.4-46.2$ & $0.5-20.2$ \\
$\geq 10 \%$ 10-year CVD risk & $266(23.6)$ & $253(33.1)$ & $13(3.6)$ \\
Current aspirin use & $44(3.9)$ & $34(4.5)$ & $10(2.8)$ \\
$\quad$ Appropriate & $16(36)$ & $15(44)$ & $1(10)$ \\
Inappropriate & $28(64)$ & $19(56)$ & $9(90)$ \\
\hline
\end{tabular}

Data are numbers (\%). CVD: cardiovascular disease

Table 3. The 10-year CVD risk and aspirin use according to gender and age

\begin{tabular}{|c|c|c|c|c|c|c|}
\hline & \multicolumn{3}{|c|}{ Men } & \multicolumn{3}{|c|}{ Women } \\
\hline & $\begin{array}{l}\text { Total } \\
(n=764)\end{array}$ & $\begin{array}{l}\text { 50-59 years } \\
(n=583)\end{array}$ & $\begin{array}{l}\text { 60-69 years } \\
(n=181)\end{array}$ & $\begin{array}{l}\text { Total } \\
(n=361)\end{array}$ & $\begin{array}{l}\text { 50-59 years } \\
(n=229)\end{array}$ & $\begin{array}{l}\text { 60-69 years } \\
(n=132)\end{array}$ \\
\hline 10-year CVD risk (\%) & $1.4-46.2$ & $1.4-25.8$ & $4.3-46.2$ & $0.5-20.2$ & $0.5-8$ & $1.8-20.2$ \\
\hline$\geq 10 \% 10$-year CVD risk & $253(33.1)$ & 117 (20.1) & $136(75.1)$ & $13(3.6)$ & $0(0)$ & $13(9.8)$ \\
\hline Current aspirin use & $34(4.5)$ & $20(3.4)$ & $14(7.7)$ & $10(2.8)$ & $4(1.7)$ & $6(4.5)$ \\
\hline Appropriate & $15(44)$ & $5(25)$ & $10(71)$ & $1(10)$ & $0(0)$ & $0(0)$ \\
\hline Inappropriate & $19(56)$ & $15(75)$ & $4(29)$ & $9(90)$ & $4(100)$ & $6(100)$ \\
\hline
\end{tabular}

Data are numbers (\%). CVD: cardiovascular disease

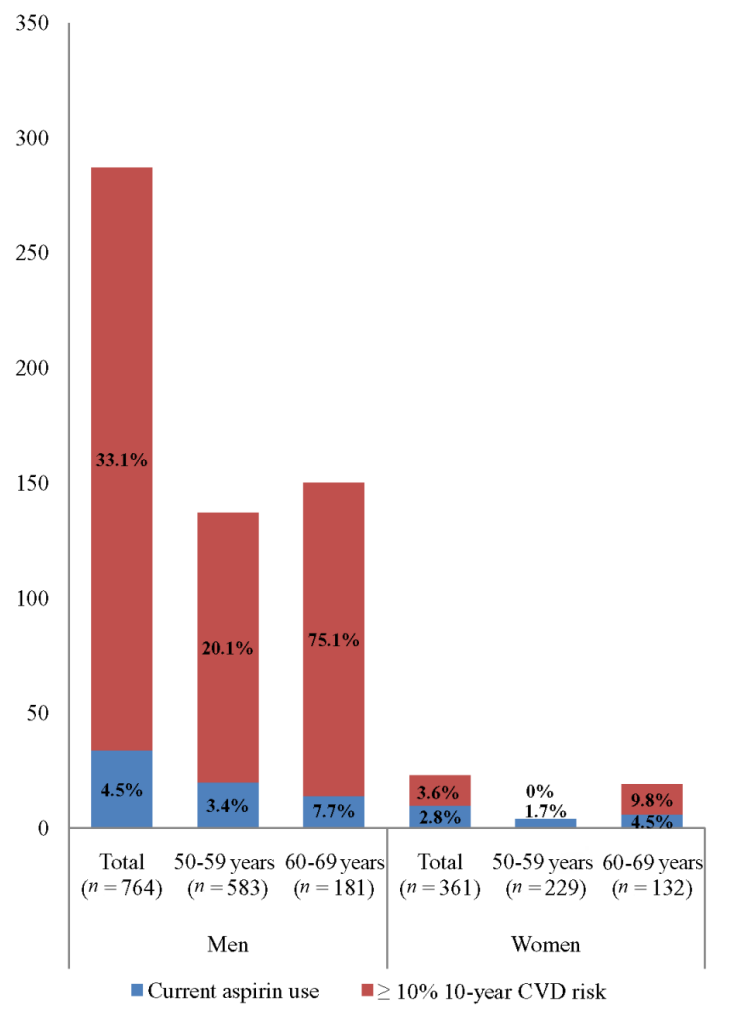

Figure 1. The 10-year cardiovascular disease (CVD) risk and the aspirin use according to gender and age

Table 4 shows the relationship between the factors of ASCVD risk calculation and taking aspirin. Treatment for hypertension was significantly associated with taking aspirin [odd ratio (OR) 7.49; 95\% confidence interval (CI) 3.62-15.49]. Though there were no significant differences, a trend toward taking aspirin was related with age (OR 1.02; 95\% CI 0.99-1.12) and men (OR 1.64; 95\% CI 0.79-3.35). Smoking was inversely related with aspirin use (OR 0.59; 95\% CI 0.24-1.42). Of the 1125 participants, 265 (23.6\%) underwent IMT measurements. The mean IMT was significantly greater in the $\geq 10 \% 10$-year CVD risk group $(n=70,0.83 \pm$ $0.13 \mathrm{~mm})$ than in the $<10 \% 10$-year CVD risk group $(n=195,0.78 \pm 0.12 \mathrm{~mm} ; P=0.003)$. 
Table 4. Logistic regression analysis for the factors of ASCVD risk calculation and taking aspirin

\begin{tabular}{lll}
\hline ASCVD risk calculation factors & OR $(\mathbf{9 5} \% \mathbf{C l})$ & $\boldsymbol{P}$ value \\
\hline Men & $1.64(0.79-3.35)$ & 0.179 \\
Age & $1.05(0.99-1.12)$ & 0.106 \\
HDL-cholesterol & $0.98(0.94-1.01)$ & 0.124 \\
Total cholesterol & $0.99(0.98-1.01)$ & 0.346 \\
Treatment for hypertension & $7.49(3.62-15.49)$ & $<0.0001^{\star}$ \\
Systolic blood pressure & $0.98(0.96-1.01)$ & 0.179 \\
Cigarette smoker & $0.59(0.24-1.42)$ & 0.236 \\
Diabetes & $1.27(0.56-2.86)$ & 0.565 \\
\hline
\end{tabular}

ASCVD: atherosclerotic cardiovascular disease; OR: odds ratio; Cl: confidence interval; HDL: high density lipoprotein. Significant $P$ is marked with *

\section{DISCUSSION}

Based on the ACC/AHA ASCVD risk estimator and the 2016 USPSTF recommendations, this study demonstrated that aspirin was indicated in $23.6 \%$ of the participants undergoing medical check-up but only $6 \%$ of the participants were taking it appropriately. These results are similar to previous findings that showed the frequency of aspirin use $\mathrm{e}^{[9-11]}$. The role of aspirin in primary prevention among individuals without known CVD is currently unclear ${ }^{[2,4,12]}$. However, high-risk patients who are not receiving aspirin are at an increased risk of CVD events. Low-risk patients are also exposed to the adverse bleeding risk with unnecessary use of aspirin. For primary prevention of CVD, decisions regarding aspirin use should be highly individualized ${ }^{[13]}$. An alternative approach that may be helpful in determining the risk and benefit from aspirin therapy is using a risk assessment tool. It is helpful that healthcare providers will be able to estimate the CVD risk for an individual patient.

In this study, logistic regression analysis revealed that though SBP did not have any effect on the aspirin use (OR 0.98; 95\% CI 0.96-1.01), treatment for hypertension was strongly associated with taking aspirin. It might be related with doctor's coprescription of aspirin with antihypertensive drugs in outpatient clinic, suggesting that there may be an opportunity for decreasing the rate of CVD as well as the risk for major bleeds through tailored education for physicians on aspirin use. Our study also showed that there was a trend toward taking aspirin with men and aging. Advancing age is a well-known non-modifiable risk factor for CVD. The cumulative effects of aging substantially increase the CVD risk, but the burden of CVD risk can be reduced in part by the modification of traditional risk factors ${ }^{[14]}$. A higher frequency of diabetes and smoking in men may be associated with these results as well. Potentially modifiable risk factors, such as hypertension, diabetes, dyslipidemia, tobacco use, and physical inactivity, account for most of the risk of $\mathrm{CVD}^{[15,16]}$. Medications to control blood pressure and lipids, smoking cessation, diet, and exercises are the interventions broadly applicable to the general population. There is another chance for decreasing the rate of CVD through personalized education for individuals on modifiable risk factors. The optimization of CVD prevention for individuals can identify and achieve the control of risk factors safely, expeditiously, and costeffectively.

In our study, the mean IMT was significantly greater in the $\geq 10 \% 10$-year CVD risk group than in the $<10 \%$ 10-year CVD risk group. Several longitudinal studies have demonstrated that an increased carotid IMT can have an independent, synergistic risk prediction power for stroke and $\mathrm{MI}^{[17]}$. While a carotid ultrasonography screening policy is not warranted in the general population, it might be considered in subjects with a higher 10 -year CVD risk to better stratify their actual risk ${ }^{[18,19]}$. Further studies are required to address the role of carotid ultrasonography in primary prevention of vascular events in high-risk subjects.

There were several limitations in this study. The most important limitation was that aspirin use was determined based on a self-report and this might have led to an underestimation of the actual use. The socioeconomic status was not determined and this could have resulted in overestimation of the number of individuals in 
the population taking aspirin. Due to the small sample size, we could not evaluate the factors regarding to the inappropriate use of aspirin. Only 3.9\% of participants were taken aspirin regularly in this study. Finally, this trial enrolled only Korean participants, limiting the generalizability of our findings to other geographic regions. Concerns have been raised that this guideline is the only US-based, externally validated equations that report risk as a combination of CVD, stroke, and CRC events. There may be limitations in general applications of the risk functions to other ethnic populations due to the differences in diet pattern, life style, social environment, or genetic predisposition ${ }^{[20,21]}$. These limitations should be considered during the interpretation of our data.

In conclusion, the decision to take aspirin is still an individual one, which should be made after careful evaluation of the trade-off between the benefits and risks, particularly the risk of major bleeding. This study showed that aspirin would be indicated in nearly one-quarter of the adults aged 50 to 69 years who undergo a medical check-up but only 6\% were taking aspirin appropriately based on the 2016 USPSTF recommendations. These results suggested that there may be an opportunity for decreasing the rate of CVD as well as the risk for major bleeds through tailored education on aspirin use.

\section{DECLARATIONS}

\section{Authors' contributions}

Conceived of the study: Baik JS

Recruitment and clinical assessment: Im JH, Han SW, Lee SY

Statistical analysis: Han SW

Drafted the initial version of the report: Im JH

Revision and editing of the report: all authors

\section{Data source and availability}

The dataset used and analysed during the current study are available from the corresponding author on reasonable request.

\section{Financial support and sponsorship}

None.

\section{Conflicts of interest}

There are no conflicts of interest.

\section{Patient consent}

All study participants provided informed consent.

\section{Ethics approval}

The study design was approved by the appropriate ethics review board and conducted in accordance with the Good Clinical Practice guidelines and the Declaration of Helsinki.

\section{Copyright}

(c) The Author(s) 2018.

\section{REFERENCES}

1. US Preventive Services Task Force. Aspirin for the prevention of cardiovascular disease: US Preventive Services Task Force recommendation statement. Ann Intern Med 2009;150:396-404.

2. Ittaman SV, VanWormer JJ, Rezkalla SH. The role of aspirin in the prevention of cardiovascular disease. Clin Med Res 2014;12:147-54. 
3. Xie M, Shan Z, Zhang Y, Chen S, Yang W, Bao W, Rong Y, Yu X, Hu FB, Liu L. Aspirin for primary prevention of cardiovascular events: meta-analysis of randomized controlled trials and subgroup analysis by sex and diabetes status. PLoS One 2014;9:e90286.

4. Bibbins-Domingo K; US Preventive Services Task Force. Aspirin use for the primary prevention of cardiovascular disease and colorectal cancer: US Preventive Services Task Force recommendation statement. Ann Intern Med 2016;164:836-45.

5. Antithrombotic Trialists' (ATT) Collaboration; Baigent C, Blackwell L, Collins R, Emberson J, Godwin J, Peto R, Buring J, Hennekens C, Kearney P, Meade T, Patrono C, Roncaglioni MC, Zanchetti A. Aspirin in the primary and secondary prevention of vascular disease: collaborative meta-analysis of individual participant data from randomised trials. Lancet 2009;373:1849-60.

6. Expert Committee on the Diagnosis and Classification of Diabetes Mellitus. Report of the expert committee on the diagnosis and classification of diabetes mellitus. Diabetes Care 2003;26 Suppl 1:S5-20.

7. Touboul PJ, Hennerici MG, Meairs S, Adams H, Amarenco P, Bornstein N, Csiba L, Desvarieux M, Ebrahim S, Hernandez Hernandez R, Jaff M, Kownator S, Naqvi T, Prati P, Rundek T, Sitzer M, Schminke U, Tardif JC, Taylor A, Vicaut E, Woo KS. Mannheim carotid intima-media thickness and plaque consensus (2004-2006-2011). An update on behalf of the advisory board of the 3rd, 4th and 5th watching the risk symposia, at the 13th, 15th and 20th European Stroke Conferences, Mannheim, Germany, 2004, Brussels, Belgium, 2006, and Hamburg, Germany, 2011. Cerebrovasc Dis 2012;34:290-6.

8. Kim SN, Lee HS, Nam HS, Lee HR, Kim JM, Han SW, Park JH, Baik JS, Kim JY. Carotid intima-media thickness is inversely related to bone density in female but not in male patients with acute stroke. J Neuroimaging 2016;26:83-8.

9. Hira RS, Kennedy K, Nambi V, Jneid H, Alam M, Basra SS, Ho PM, Deswal A, Ballantyne CM, Petersen LA, Virani SS. Frequency and practice-level variation in inappropriate aspirin use for the primary prevention of cardiovascular disease: insights from the National Cardiovascular Disease Registry's Practice Innovation and Clinical Excellence registry. J Am Coll Cardiol 2015;65:111-21.

10. Mainous AG, Tanner RJ, Shorr RI, Limacher MC. Use of aspirin for primary and secondary cardiovascular disease prevention in the United States, 2011-2012. J Am Heart Assoc 2014;3:e000989.

11. Manes C, Giacci L, Sciartilli A, D’Alleva A, De Caterina R. Aspirin overprescription in primary cardiovascular prevention. Thromb Res 2006;118:471-7.

12. Miedema MD, Huguelet J, Virani SS. Aspirin for the primary prevention of cardiovascular disease: in need of clarity. Curr Atheroscler Rep 2016;18:4.

13. Mora S, Ames JM, Manson JE. Low-dose aspirin in the primary prevention of cardiovascular disease: shared decision making in clinical practice. JAMA 2016;316:709-10.

14. Dhingra R, Vasan RS. Age as a risk factor. Med Clin North Am 2012;96:87-91.

15. Yusuf S, Hawken S, Ounpuu S, Dans T, Avezum A, Lanas F, McQueen M, Budaj A, Pais P, Varigos J, Lisheng L. Effect of potentially modifiable risk factors associated with myocardial infarction in 52 countries (the INTERHEART study): case-control study. Lancet 2004;364:937-52.

16. Cannon CP. Cardiovascular disease and modifiable cardiometabolic risk factors. Clin Cornerstone 2007;8:11-28.

17. Touboul PJ, Labreuche J, Vicaut E, Amarenco P. Carotid intima-media thickness, plaques, and Framingham risk score as independent determinants of stroke risk. Stroke 2005;36:1741-5.

18. Prati P, Tosetto A, Vanuzzo D, Bader G, Casaroli M, Canciani L, Castellani S, Touboul PJ. Carotid intima media thickness and plaques can predict the occurrence of ischemic cerebrovascular events. Stroke 2008;39:2470-6.

19. Jonas DE, Feltner C, Amick HR, Sheridan S, Zheng ZJ, Watford DJ, Carter JL, Rowe CJ, Harris R. Screening for asymptomatic carotid artery stenosis: a systematic review and meta-analysis for the US Preventive Services Task Force. Ann Intern Med 2014;161:336-46.

20. Bineau S, Dufouil C, Helmer C, Ritchie K, Empana JP, Ducimetiere P, Alperovitch A, Bousser MG, Tzourio C. Framingham stroke risk function in a large population-based cohort of elderly people: the 3C study. Stroke 2009;40:1564-70.

21. Jee SH, Park JW, Lee SY, Nam BH, Ryu HG, Kim SY, Kim YN, Lee JK, Choi SM, Yun JE. Stroke risk prediction model: a risk profile from the Korean study. Atherosclerosis 2008;197:318-25. 